\title{
ORIGIN OF CENTRES INVOLVED IN BLUE AND ORANGE LUMINESCENCE OF $6 \mathrm{H}-\mathrm{SiC}^{*}$
}

\author{
A. Wysmolek, J.M. Baranowski and M. KamińSKa \\ Institute of Experimental Physics, Warsaw University \\ Hoża 69, 00-681 Warszawa, Poland
}

\begin{abstract}
We present new results of luminescence of $n$-type $6 \mathrm{H}$-SiC crystals. We have found two shallow donors with ionization energies at $60 \mathrm{meV}$ and $140 \mathrm{meV}$. We have shown that the blue luminescence is not affected by the ionization of the shallower donor and is related to deeper donor which we attribute to $\mathrm{N}$ at $\mathrm{C}$-site. We propose that the origin of the more shallower donor at $60 \mathrm{meV}$ is related to carbon vacancy. We have found that the intensity of the orange luminescence increases under infrared illumination. This result confirms that the orange luminescence is due to conduction band-deep centre transitions. We believe that deep centre responsible for the orange luminescence is the silicon vacancy.
\end{abstract}

PACS numbers: 78.55.-m, 78.20.-e, 78.60.Kn

\section{Introduction}

The characteristic property of $6 \mathrm{H}-\mathrm{SiC}$ is a presence of blue and orange luminescence bands. The blue band has been previously attributed to donor-acceptor pair (D-A) transitions involving shallow donors related to nitrogen occupying hexagonal and quasi-cubic carbon lattice sites [1]. Ionization energies of these donors were determined as $81.0 \mathrm{meV}$ for hexagonal site, $137 \mathrm{meV}$ and $142 \mathrm{meV}$ for two inequivalent cubic sites respectively [2]. These large site dependent energy differences of the donor ground state are difficult to explain within the effective-mass theory.

The nature of centres responsible for the orange band is not known but it was early proposed that it originates from both donor-deep defect and conduction band-deep defect transitions [3].

*This work was partially supported by the State Committee for Scientific Research (Republic of Poland) grant No. PB0334/P4/94/06. 


\section{Experiment}

We have studied $n$-type $6 \mathrm{H}-\mathrm{SiC}$ crystals grown by Lely [4] method unintentionally doped with $\mathrm{N}$ which is a donor and intentionally doped with $\mathrm{Al}$ which is an acceptor. Photoluminescence (PL) measurements were performed within the spectral region between $1.5 \mathrm{eV}$ and $3.1 \mathrm{eV}$ using Spex 500M monochromator equipped with photomultiplier. PL excitation above band gap was performed by $3.8 \mathrm{eV}$ line of $\mathrm{He}-\mathrm{Cd}$ laser.

In the case of PL measurements under additional infrared (IR) illumination, halogen lamp with silicon filter was used. To measure an influence of the infrared illumination on the PL intensity two experimental methods were applied. In the first case PL measurements have been performed with and without continuous IR illumination using photon counting system. In the second method PL intensity changes were detected by means of lock-in amplifier modulating IR radiation and using it as reference signal. The results obtained from both two methods are equivalent.

\section{Results and discussion}

Thermoluminescence measurements performed for the orange band showed peaks at $30 \mathrm{~K}$ and $70 \mathrm{~K}$ [3]. They were attributed to ionization of donors with estimated energies at $60 \mathrm{meV}$ and $140 \mathrm{meV}$. The EPR investigation [4] revealed that the $140 \mathrm{meV}$ donor is due to the nitrogen impurity at carbon site.

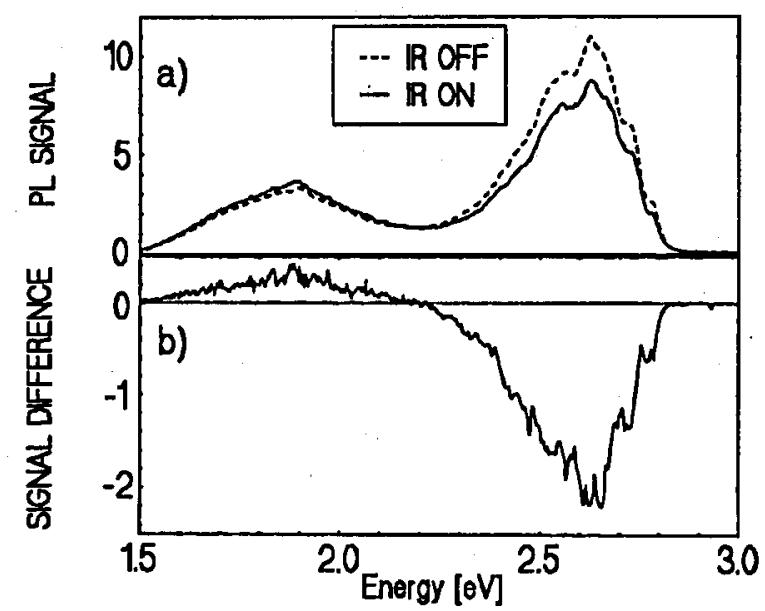

Fig. 1. Photoluminescence spectra of $n$-type $6 \mathrm{H}$-SiC (a) measured at $4.2 \mathrm{~K}$ with (solid line) and without (dashed line) additional infrared illumination. The change of luminescence induced by additional infrared radiation (b). 


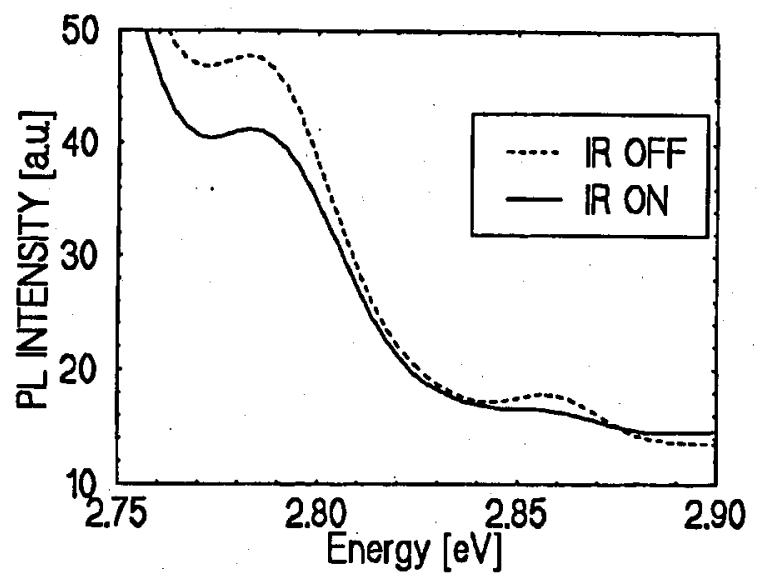

Fig. 2. The region of zero phonon and hot line of the PL spectra obtained at $85 \mathrm{~K}$ with (solid line) and without (dashed line) additional infrared illumination.

The involvement of $\mathrm{N}_{\mathrm{C}}$ impurity in the blue luminescence has been directly confirmed by the study of the zero phonon hot line (see Fig. 1) which is separated from the zero phonon luminescence line by about $65 \mathrm{meV}$ which is in good agreement with the $60.3 \mathrm{meV}$ splitting of the $\mathrm{N}_{\mathrm{C}}$ donor ground state obtained from Raman scattering experiments [5]. This interpretation was confirmed by the results of PL measurements performed under IR illumination (see Fig. 1 and Fig. 2). The hot line was suppressed by IR radiation in the same way as the rest of the blue band. It suggests that previous interpretation [1] of the hot line as a conduction band-acceptor transition should be revised, because the intensity of such luminescence should not be suppressed by donor ionization.

Investigations of the blue band luminescence performed at wide range of temperatures showed that the intensity and the shape of this band have practically not changed between $4.2 \mathrm{~K}$ and $70 \mathrm{~K}$. This means that the blue band is not affected by $60 \mathrm{meV}$ donor ionization. The EPR experiments [4] have shown that only $140 \mathrm{meV}$ donor is related to nitrogen centre and the $60 \mathrm{meV}$ donor has to have some other origin. Taking into account these results, we came to the conclusion that only $140 \mathrm{meV} \mathrm{N} \mathrm{C}^{-} \mathrm{Al}$ acceptor pairs are responsible for the blue luminescence. The question arises why more shallow $60 \mathrm{meV}$ donor does not form pairs with $\mathrm{Al}$ acceptors. The apparent lack of overlap between the $60 \mathrm{meV}$ donor and $\mathrm{Al}$ acceptor wave function may be explained by a strong localization of the donor state. A structural defect such as vacancy may form a localised state. Theoretical studies of formation energies and the electronic structure of native defects in cubic $\mathrm{SiC}[6]$ suggest that a state formed from silicon dangling bond thus the carbon vacancy may be a good candidate for a relatively shallow localised donor.

Moreover, the assumption that only $140 \mathrm{meV}$ donor is involved in the blue band luminescence simplifies the previous [1] interpretation of its spectral structure. The model with zero phonon lines related to nitrogen donors with ionization energies at $81 \mathrm{meV}, 137 \mathrm{meV}$ and $142 \mathrm{meV}[1,2]$ may be replaced by a model in 
which only one zero phonon line with TA and LO phonon replicas is taken into account. Such interpretation explains the temperature behaviour of the blue band described in the previous section.

Photoluminescence experiments showed the increase in the intensity of the orange band under IR (see Fig. 1). Thus, increase in free carrier concentration in the conduction band leads to increase in the orange luminescence which suggests that the corresponding optical transitions are related to conduction band-deep centre. A good candidate to a deep centre seems to be the silicon vacancy [6]. In such centre the wave function is formed from deep carbon dangling bonds and may be expected that it is a deep centre.

We believe that the involvement of structural defects such as carbon and silicon vacancies in the optical transitions in a natural way explains presence of the blue and orange luminescence in $\mathrm{SiC}$ and clears up a controversy connected with these bands.

\section{Acknowledgments}

We are very grateful to Dr. S. Vlaskina from Institute of Semiconductors Physics, Ukrainian Academy of Sciences, for supplying us with SiC crystals.

\section{References}

[1] M. Ikeda, H. Matsunami, T. Tanaka, J. Lumin. 20, 111 (1979).

[2] W. Suttrop, G. Pensl, W.J. Choyke, R. Stein, S. Leibenzader, J. Appl. Phys. 72, 3708 (1992).

[3] A. Wysmolek, P. Mroziński, R. Dwiliński, S. Vlaskina, M. Kamińska, Acta Phys. Pol. A 87, 437 (1995).

[4] B. Suchanek, R. Dwiliński, M. Kamińska, M. Palczewska, S. Vlaskina, Acta Phys. Pol. A 87, 321 (1995).

[5] P.J. Colwell, M.V. Klein, Phys. Rev. B 6, 498 (1972).

[6] C. Wang, J. Bernholc, R.F. Davis, Phys. Rev. B 38, 12752 (1988). 